\title{
Adaptive Channel Selection Through Collaborative Sensing
}

\author{
Guang-Hua Yang ${ }^{\star}$, Haitao Zheng ${ }^{\dagger}$, Jun Zhao ${ }^{\ddagger}$ and Victor O. K. $\mathrm{Li}^{\star}$ \\ ${ }^{\star}$ Dept. of Elec. \& Electronic Engineering, Univ. of Hong Kong, Hong Kong, China, \{ghyang, vli\}@eee.hku.hk \\ ${ }^{\dagger}$ Dept. of Computer Science, Univ. of California, Santa Barbara, CA, U.S.A, htzheng@cs.ucsb.edu \\ ${ }^{\ddagger}$ Wireless \& Networking Group, Microsoft Research Asia, Beijing, China, junzhao@microsoft.com
}

\begin{abstract}
Proper channel selection is essential to exploit the benefits of multi-channel systems by distributing conflicting transmissions across non-interfering channels. Critical to channel selection is the channel quality metric. We propose a busy time ratio $(B T R)$ metric that captures channel contention and user traffic load under a variety of network dynamics. We also propose a distributed collaborative sensing scheme to reduce sensing overhead and energy consumptions. The proposed algorithms can be implemented using conventional 802.11 hardware with single radio interface. The proposed metric can be integrated with routing and channel selection. Experimental results show that the proposed scheme significantly outperforms the existing channel selection methods.
\end{abstract}

Index Terms-Multiple channel, channel selection, available bandwidth, busy time ratio

\section{INTRODUCTION}

A critical problem in multi-hop wireless networks is throughput degradation due to interference among multiple simultaneous transmissions. Multi-channel systems were introduced to alleviate interference by distributing interfering links to non-interfering channels. For dense, heavily loaded systems, the number of potentially conflicting links outnumbers the number of channels, and a good channel selection algorithm is essential to system performance. The problem is exacerbated by the sporadic nature of multi-hop wireless networks, in particular node mobility, fragile links, traffic dynamics.

Critical to a channel selection scheme is the metric to characterize channel quality. Prior efforts have proposed to use link signal-to-noise ratio [1], or probed packet delay [2] as channel metrics. While providing a good estimation of channel quality for point to point links, these metrics do not accurately characterize channel contentions. The work in [3], [4], [5] approximates contention through the number of competing links or traffic volume, ignoring the impact of heterogenous traffic pattern and heterogeneous link quality across links. These motivate the search for a channel metric that can properly characterize channel utilization under a variety of traffic and topology dynamics and time-varying channel impairments.

The metric design is also constrained by the complexity and overhead of measurement techniques. We consider a distributed multi-hop wireless network without central management. We assume commonly available 802.11 devices, each

This research is supported in part by the Areas of Excellence Scheme established under the University Grants Committee of the Hong Kong Specia Administrative Region, China (Project No. AoE/E-01/99). Part of this work was done while Guanghua Yang was a visiting student in Microsoft Research Asia. equipped with a half-duplex single radio interface. For this type of network, we propose a device-centric channel selection approach where each device senses channel conditions and adapts its channel usage to network and traffic dynamics. In particular, we propose a simple, busy time ratio $(B T R)$ based channel metric, and a set of collaborative sensing techniques that trade off complexity with accuracy. The proposed approach allows each user to collect information on the quality of multiple channels without exhaustively accessing all the channels, making channel sensing energy-efficient. We also incorporate the $B T R$ metric into routing and channel selection for throughput improvement in multi-hop transmissions. Extensive experimental results demonstrate the effectiveness of the proposed metric and sensing techniques.

The rest of the paper is organized as follows. In Sec. II, we briefly introduce related work in channel quality measurements. In Sec. III, we present the BTR-based channel metric and propose several collaborative sensing techniques to estimate $B T R$ in IEEE 802.11 DCF systems. In Sec. V, we propose a $B T R$-based adaptive channel and route selection framework. The advantages of the proposed metric and framework are demonstrated by experimental results in Sec. VI. Finally, we conclude in Sec. VII.

\section{RELATED WORK}

In this section, we briefly overview existing channel evaluation methods and outline the research problem.

The simplest approach is to ignore channel quality and randomly select channels [6]. Under contention fluctuations and time-varying channel impairments, this approach is obviously not optimal. The work in [2] applies channel probing, i.e., sending probing packets over the air to estimate channel bandwidth and delay. This approach may heavily stress the communication resources of bandwidth- or energy-constrained devices. In addition, injecting extra traffic could result in excessive contention and obligatory backoff, which lead to system throughput degradation.

On the other hand, on-line signal based channel estimation provides an inexpensive alternative to channel probing. The work in [1] measures signal to interference plus noise ratio (SINR), indirectly estimating the maximum throughput a user can get without any contention. However, it fails to account for the impact of user contention. The work in [3], [4] use the number of competing links $N$ to characterize the level of contention. However, the estimation is subject to the assumption of homogeneous traffic which is not commonly 
observed. Other studies have shown that estimation of $N$ suffers from non-negligible estimation errors [7], [8], and a high computational complexity [3].

The work in [5] uses the aggregated traffic served by a channel to represent its quality. However, it does not consider the impact of user-dependent channel impairments; and channel usage due to packet retransmissions. In addition, this approach assumes each user can successfully collect traffic information from neighbors in close proximity, making the measurement sensitive to the reliability of message exchanging, and the level of user cooperation.

\section{iII. Busy Time Ratio as Channel Selection Metric}

Let available bandwidth $B_{\text {avail }}$ represent the maximum bandwidth a channel can provide to a new link. Intuitively, a user tends to select the channel with the highest $B_{\text {avail }}$. We can approximate $B_{\text {avail }}$ as the difference between the maximum saturated bandwidth of the channel and the total bandwidth occupied by existing links. Assuming each device performs CSMA/CA [9], we can derive $B_{\text {avail }}$ by estimating mean frame size (MFS) and eavesdropping on the network allocation vector ( $N A V)$ [10]. However, the $N A V$ information collected by a device only accounts for the link transmissions within a device's transmission range. Instead, channel quality depends on the level of transmissions within a node's interference range. In general the interference range is much larger than the transmission range, e.g. by a factor of 2. Hence, $N A V$-based available bandwidth under-estimates channel usage.

We propose to replace $N A V$ with busy time ratio (BTR). $B T R$ is defined as the total time that the physical channel is busy normalized by the measurement time,

$$
B T R=\frac{\text { Total busy time }}{\text { Total measurement time }} .
$$

Following a set of derivations similar to [10], we can derive $B_{\text {avail }}$ from $B T R$ and MFS by solving a set of nonlinear equations. To verify the accuracy of the proposed approach, we perform a set of experiments comparing the analytical results to the actual measurements. We randomly set up a set of links in a given area, each carrying random traffic with the same packet size but different packet rate. We analytically compute $B_{\text {avail }}$ based on the measured BTR at each node. In Fig. 1, this derivation is compared to the actual throughput each link obtains. We see that the estimation is fairly accurate under fixed frame size $M F S$. There exists a consistent linear relation between $B_{\text {avail }}$ and $B T R$ for a fixed $M F S$.

While algorithms exist to estimate $B_{\text {avail }}$ from the observed $M F S$ and $B T R$, they are computationally complex [10] and may heavily stress the energy-constrained devices. Motivated by the consistent linear trend in Fig. 1, we propose to directly use $B T R$ as the channel metric. The same figure also suggests that MFS can heavily impact the available bandwidth for a given $B T R$. However, good estimation of MFS requires excessive sensing and reliable decoding of all packets, which is infeasible for energy-constrained devices. As a result, we ignore the impact of $M F S$, trading complexity with accuracy. We examine the related estimation error in Sec. VI-B, which confirms that the impact is small in the simulated networks.

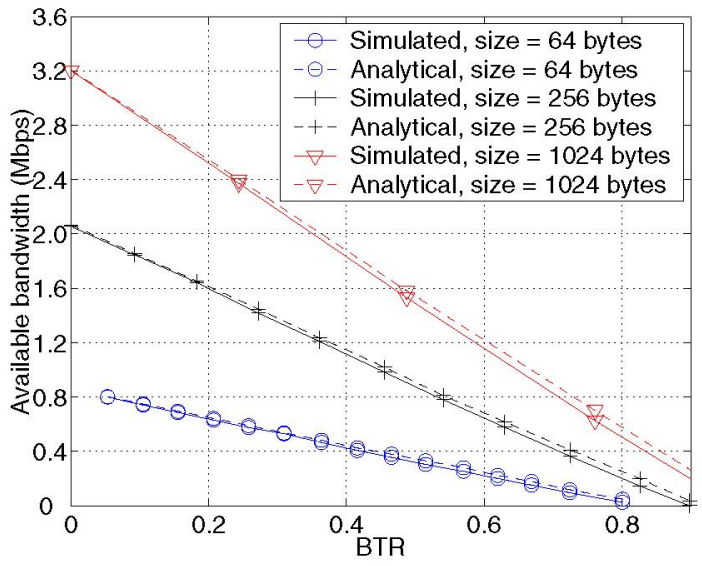

Fig. 1. Comparison of $B_{\text {avail }}$ estimated on $M F S$ and $B T R$ with that obtained from simulations. $M F S=64,256,1024$ bytes respectively.

While the proposed $B T R$ metric focuses on characterizing user contentions, it also captures variations in user link quality. A user experiencing bad connections consumes additional time by using lower rates and extra retransmissions. In this work, we assume that each user experiences statistically uniform impairments (path loss, shadowing and fading) across all the channels; i.e. the impairments are frequency non-selective. Therefore, BTR directly indicates the channel usage and the available bandwidth for each new link. For time-varying frequency-selective fading, we can extend $B T R$ to account for average channel signal-to-noise ratio by periodically probing each channel. Overall, BTR provides a good approximation of channel quality, taking into account the impact of user contentions, traffic heterogeneity, transmission failures and retransmissions.

\section{Collaborative BTR Measurement}

In this section, we provide a measurement scheme that allows each user to observe BTRs of multiple channels without having to sense each channel individually. In particular, each device performs local measurement on the channel it is currently using, and utilizes a collaborative sensing scheme to accumulate measurements on other channels.

\section{A. Local BTR Sensing}

Each device measures the $B T R$ of the channel it is currently using. There are two measuring schemes that trade off complexity with precision.

\section{a) Physical measurement}

This scheme invokes the carrier-sensing module to measure the power level of the received signal. When the power level exceeds a pre-defined threshold, the channel is busy. Given the carrier-sensing module is built-in, this scheme is simple to implement. However, since sensing consumes similar power as transmissions, this leads to excessive energy consumption. We refer to the physically measured $B T R$ as $B T R_{P H Y}$.

\section{b) Virtual measurement}

To reduce energy consumption due to carrier sensing, each node can estimate $B T R$ by eavesdropping on MAC control messages. In particular, $N A V$ information embedded in MAC 
frame headers approximates the duration of the current transmission. Each device can obtain a good estimation of $B T R$ by accumulating self-transmission time, and $N A V$-specified neighboring links' transmission time as well as protocolspecific overhead. This measurement significantly improves energy efficiency - during the time declared by neighbor's $N A V$ signals, a device can configure its radio to a lowpower dozing mode [11]. We refer to the measured BTR as $B T R_{M A C}$.

Fig. 2 illustrates the time-line of both measurement schemes. The busy/idle states represent physical sensing results of Node 1 using physical measurement. The $N A V$ related blocks represent the channel busy durations estimated at Nodes $1-6$ by using virtual sensing. It should be noted that virtual measurement requires successful decoding of $N A V$ signals, and can therefore only detect transmissions within its transmission range, rather than interference range. This leads to under-estimation of channel usage. Next we propose a collaboration-based approach to overcome this problem.

\section{B. Collaborative Sensing}

While virtual sensing scheme provides an energy-efficient alternative to physical sensing, its estimation accuracy is limited by the difference between the interference range and the transmission range. In addition, the sensing complexity and overhead scales linearly with the number of channels. In this section, we propose a collaborative sensing scheme where devices can obtain an accurate estimation of $B T R$ of multiple channels without physically scanning and carrier-sensing all of them. The scheme is motivated by the fact that users in close proximity observe similar channel usage.

The detailed procedure is as follows. Each device conducts virtual sensing on its current channel $i$ in use, to obtain a selfobserved $B T R_{M A C}^{S}(i)$. Each device periodically broadcasts this measurement to its $k$-hop neighbors, where

$$
k=\left\lfloor\frac{\text { Interference Range }}{\text { Transmission Range }}\right\rfloor .
$$

The $B T R$ broadcast can be embedded into regular control messages, communicated through a predefined coordination channel, or during a synchronized coordination time slot [4], [14]. Each device collects the $B T R$ broadcasts and records them in $\overrightarrow{B T R_{M A C}^{N}(.)}$ for each channel. Utilizing self and neighbor $B T R$ measurements, each device updates its $B T R$ estimation as follows:

a) For its current channel $i$ in use,

$$
B T R(i)=\max \left(B T R_{M A C}^{S}(i), \max \left(\overrightarrow{B T R_{M A C}^{N}(i)}\right)\right) .
$$

b) For other channels $j(j \neq i)$,

$$
B T R(j)=\max \left(\overrightarrow{B T R_{M A C}^{N}(j)}\right) .
$$

The proposed collaborative sensing provides a fair level of robustness against loss of $B T R$ broadcast packets. Prior work [5] estimates the traffic volume on each channel by accumulating the traffic information from each individual user. Any packet loss will lead to inaccurate estimation. For the proposed approach, $B T R$ is a local measure, and thus the contents of the $B T R$ broadcast each device receives is highcorrelated. The BTR estimation suffers only if the broadcast message from the user observing the highest BTR is lost. Such redundancy provides robustness to packet loss. We will examine this property through experimental results in Sec. VID.

\section{BTR-BAsed Channel and Routing Assignment}

In this section, we incorporate $B T R$-based channel metric into channel and route selection for multi-channel ad hoc networks. We start from single hop transmissions where link pairs use $B T R$ to negotiate a data channel. We then show that for multi-hop transmissions, BTR can be integrated into routing decisions to discover the best route and coordinate channel usage along the route. For simplicity, we assume that the MAC protocol provides a control channel or time frame for users to exchange negotiation information.

\section{A. Adaptive Channel Selection for Single-hop Transmission}

In fully connected networks, any two nodes observe the same channel status, and thus the same BTR. Hence, one node can decide the best channel to use based on self-observations. For multi-hop networks, two ends of the transmission may observe different $B T R$ on each channel, and need to negotiate the data channel selection. We propose to combine the $B T R$ of a link pair (node $u$ and $v$ ) as follows:

$$
\operatorname{BTR}(n)=\max \left\{B T R_{u}(n), B T R_{v}(n)\right\},
$$

where $n$ is the channel index. Then the channel selection is reduced to finding the channel with the lowest combined $B T R$, i.e.,

$$
n^{*}=\operatorname{argmin}_{n} \operatorname{BTR}(n) .
$$

Before a pair of users $u$ and $v$ start communications, both perform collaborative sensing to collect BTRs, and select the best channel according to (5). During transmissions, they continue to collect BTRs and adapt their channel selection to network dynamics. The decision of channel switching needs to account for not only the BTRs on other channels, but also the impact of traffic variations by moving self-traffic to the new channel. Additional mechanisms are required to avoid concurrent switching where a few node pairs (in close proximity) observe a channel with low $B T R$ and switch to it concurrently. We propose a random delay based approach where a node pair defers its channel switch by a random time interval. During this period, they keep sensing the channel and if a substantial increase in BTR is detected, the switch is canceled.

Fig. 3 illustrates an example network with two channels. A line exists between two nodes if they are within transmission range of each other. For illustration, we only show the five nodes in the middle of the network. The left table shows the BTRs of both channels observed by each node (through collaborative virtual sensing). When Node 1 and Node 2 want to start a transmission, they select channel 2 following (5).

\section{B. Route and Channel Selection in Multi-hop Networks}

The proposed $B T R$ metric can be integrated with route selection for multi-hop transmissions. We use a simple routing and channel usage strategy to illustrate the usage of $B T R$. We assume that the nodes on each route use the same channel to 


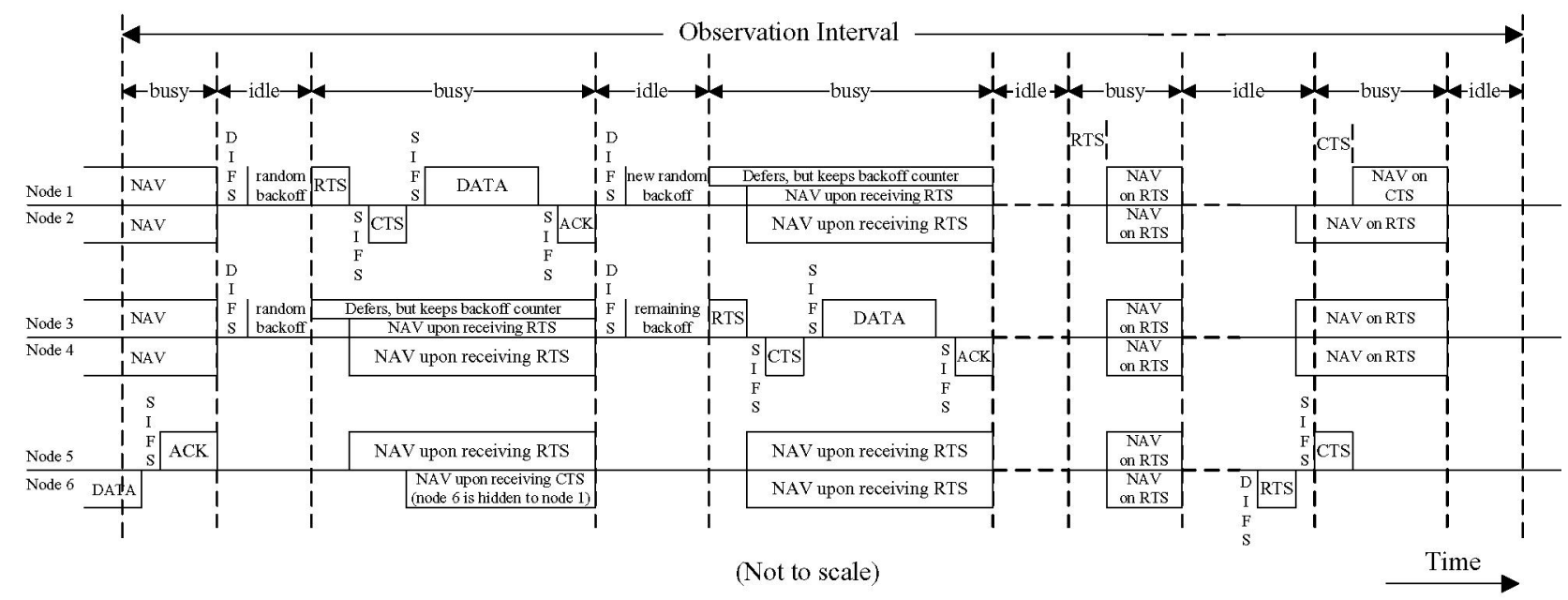

Fig. 2. $B T R$ measurement in IEEE 802.11 DCF.

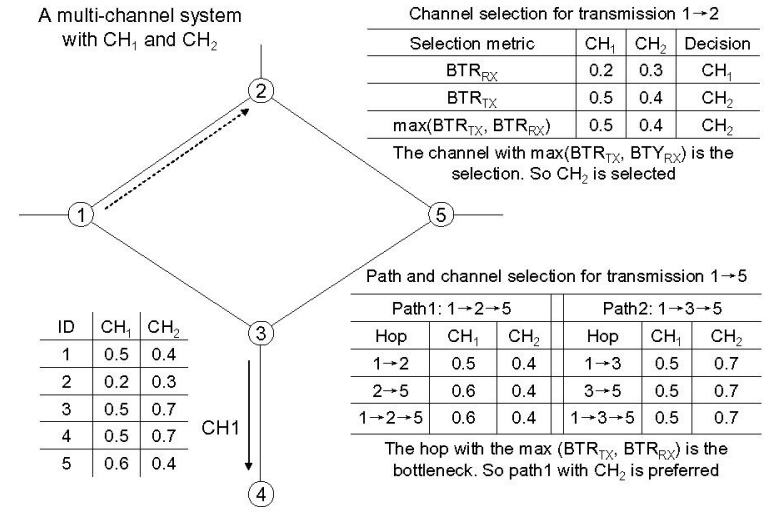

Fig. 3. An example of $B T R$-based channel and route selection.

avoid frequent channel switches. Using the example in Fig. 3, we illustrate the procedures to select the routing path and the channel to use on each hop for a transmission from Node 1 to Node 5 . In this case, there are two candidate routing paths. Each path selects the channel that minimizes the path $B T R$, defined as the maximum BTR of all the hops on the path. The bottom right table in the same figure illustrates the $B T R$ of different hops. In this case, path $1 \rightarrow 2 \rightarrow 5$ with channel 2 has the minimum path $B T R$, and thus is selected as the route.

\section{Vi. Performance Evaluation}

In this section, we conduct experimental simulations to evaluate the performance of the proposed channel selection schemes. We start from a fully connected network and compare the performance of different channel metrics. We then consider general multi-hop networks, and examine the effectiveness of the collaborative virtual sensing, and the robustness of different metrics to broadcast errors. Finally, we compare the performance of different metrics in multi-hop networks.

\section{A. Simulation Setup}

We extend NS-2 [12] with CMU wireless extensions [13] to include a multi-channel MAC protocol, heterogeneous distrib-
TABLE I

MAIN SYSTEM PARAMETERS.

\begin{tabular}{c|c}
\hline Description & Value \\
\hline Basic rate & $2 \mathrm{Mbps}$ \\
Data rate & $11 \mathrm{Mbps}$ \\
Slot time & $20 \mathrm{us}$ \\
SIFS & $10 \mathrm{us}$ \\
DIFS & $50 \mathrm{us}$ \\
PHY header & 144 bits +48 bits \\
MAC header & 224 bits \\
RTS & 160 bits + PHY header \\
CTS & 112 bits + PHY header \\
ACK & 112 bits + PHY header \\
DATA & Data length + MAC header + PHY header \\
Carrier sensing range & $2500 \mathrm{~m}$ \\
Transmitting range & $250 \mathrm{~m}$ \\
\hline CBR packet length & Uniformly distributed in $[32,1024]$ bytes \\
CBR packet rate & Uniformly distributed in $[1,50]$ packets/sec \\
Active link number $n$ & Uniformly distributed in $[1,30]$ \\
\hline
\end{tabular}

uted MAC (HD-MAC) [14]. In HD-MAC, the transmissions are based on time-frame, which is composed of a short-period coordination time slot and a long-period data transmission time slot. In the coordination time slot, users switch to a predefined coordination channel to exchange $B T R$ information and negotiate the data channel to be used in the following data transmission time slot. Then in the data transmission time slot, users switch to the selected data channel for data transmission. Related main MAC layer parameters and the traffic parameters used in the simulation are summarized in Table I.

We examine the performance of different channel metrics, including the number of contending links $N$, aggregated throughput $\sum T h r u, B T R$ and the estimated available bandwidth $B_{\text {avail }}$. All nodes have the same transmission range of $250 \mathrm{~m}$ and interference range of $500 \mathrm{~m}$. For simplicity, we assume that each user experiences the same average signal to noise ratio on each channel.

We use the decision correctness to measure the "accuracy" of the channel metrics. Decision correctness is defined as decision correctness $=\frac{\text { Number of correct selections }}{\text { Total number of selections }}$.

A channel selection decision is "correct" if it leads to a larger 
overall system throughput than any other selection.

\section{B. Comparison of Channel Selection Metrics}

We start from a fully connected network where users observe the same contention on each channel. We randomly deploy users with traffic in a $100 \mathrm{~m} \times 100 \mathrm{~m}$ area. In each instance, a pair of nodes start communications and negotiate a channel to use; while a random number of CBR flows with random traffic exist on all the channels. The characteristics of the CBR flows are also listed in Table I. Table II summarizes the results using different metrics, averaged over 3000 instances, and assuming two channels in the system. For comparison, the decision correctness, the system throughput and the link throughput of the newly joined link are listed in Table II. We also include a normalized throughput measure (using the contending link number-based metric as the baseline) to illustrate the relative difference of different metrics.

The results show that the $B_{\text {avail }}$-based channel selection yields the best performance, especially for the new link (67\% improvement compared to $N$-based scheme). BTR-based metric leads to a slightly lower throughput ( $10 \%$ degradation) but outperforms the other two metrics, especially metric $N$. Performance degradation of the metric $\sum$ Thru is due to ignoring of channel resource consumption from user contention and packet retransmissions. The decision errors of metric $B T R$ are mainly due to ignoring MFS. The results indicates that ignoring the effects of $M F S$ leads to moderate performance degradation. Overall, we see that $B T R$ provides an accurate but low cost channel quality ranker. Note that even for $B_{\text {avail }}$, decision errors exist. This is mainly due to errors in MFS estimation, and to neglecting MFS variations due to the newly joined traffics.

\section{Effectiveness of Collaborative BTR Virtual Sensing}

The proposed collaborative virtual sensing requires information from $k$-hop neighbors to estimate $B T R$. In this section, we examine the effectiveness of collaborative virtual sensing when neighbors up to 2 hops away are involved. We randomly deploy 10 node pairs in a $500 \mathrm{~m} \times 500 \mathrm{~m}$ area, each carrying a UDP flow with CBR traffic of 512 bytes/packet and 30 packets $/ \mathrm{sec}$. The results are averaged over 3000 independent deployments. Fig. 4 compares the self-observed physical $B T R B T R_{P H Y}$, self-observed virtual $B T R B T R_{M A C}$, and the $B T R$ estimated based on 2 hop collaborative virtual sensing. From the figure, we find that the self-observed $B T R_{M A C}$ is in general lower than the self-observed $B T R_{P H Y}$, due to the difference in transmission and interference ranges. In addition, collaborative virtual sensing leads to a good estimate of the $B T R_{P H Y}$.

\section{Robustness to Broadcast Errors}

Results in the previous section show that when broadcast is error-free, $\sum T h r u$ metric performs only slightly worse than $B T R$ metric. Next, we examine the robustness of the two metrics against broadcasting errors in multi-hop environments. Broadcast message transmission is conducted between any two nodes within transmission range. Broadcasting error rate (BER) is used to indicate the error level. It is defined as the
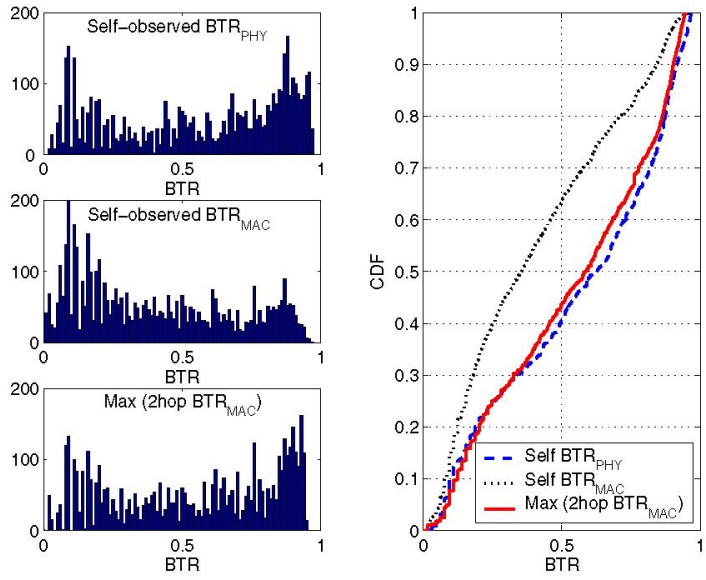

Fig. 4. Approximation of $B T R_{P H Y}$ with 2-hop neighbors' $B T R_{M A C}$. Sub-figures on the left from top to bottom are the histograms of selfobserved $B T R_{P H Y}$, self-observed $B T R_{M A C}$ and $\max \left(2 h o p B T R_{M A C}\right)$ respectively; the sub-figure on the right plots the corresponding CDFs.

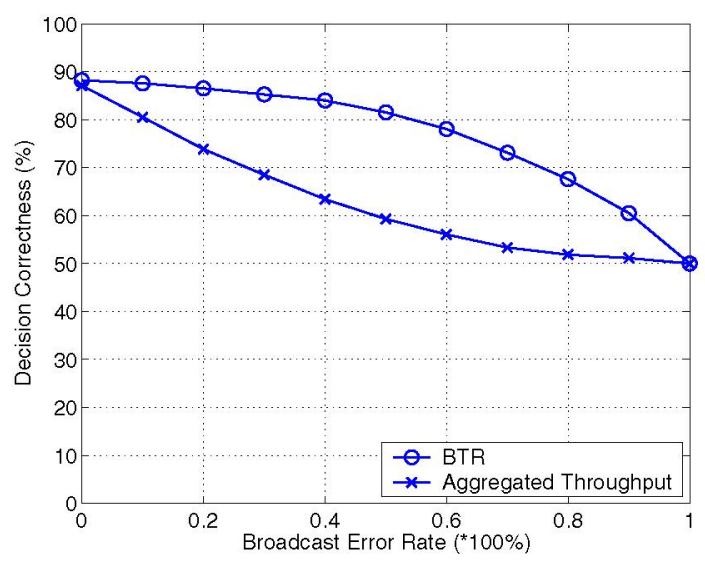

Fig. 5. Decision correctness of $B T R$ and $\sum T h r u$ based schemes under different broadcast error rates.

ratio of the number of failed broadcast message transmissions and the total number of broadcasting messages. For example, a broadcast message sent by one node to its 10 neighbors is counted as 10 broadcast messages, and if 3 of the 10 transmissions fail, then the $B E R$ is 0.3 .

We randomly deploy users in a $500 \mathrm{~m} \times 500 \mathrm{~m}$ area, and measure the decision correctness under different $B E R$. Results in Fig. 5 show that the decision correctness degrades with BER, but $B T R$ - based metric offers higher robustness. This is due to the correlation in neighboring users' $B T R$ measurements - since $B T R$ measurements taking into account the contributions of contending users in the neighborhood, users in close proximity are likely to report similar $B T R$ on each channel. Such correlations can be utilized by collaborative sensing to mitigate the effect of broadcast errors. In contrast, correct estimation of $\sum$ Thru requires reliable reception of broadcasts from neighbors.

It should be noted that users can also measure $B T R$ by physically observing each of the channels sequentially. This eliminates the dependency on message exchanging and neighbor cooperation, at the costs of a higher device power 
TABLE II

PERFORMANCE COMPARISON OF DIFFERENT CHANNEL METRICS.

\begin{tabular}{c|c||c|c||c|c}
\hline Metric & $\begin{array}{c}\text { Decision } \\
\text { Correctness }\end{array}$ & $\begin{array}{c}\text { Absolute } \\
\text { Sys. Thru. }\end{array}$ & $\begin{array}{c}\text { Normalized } \\
\text { Sys. Thru. }\end{array}$ & $\begin{array}{c}\text { Absolute Thru. } \\
\text { of New Link }\end{array}$ & $\begin{array}{c}\text { Normalized Thru. } \\
\text { of New Link }\end{array}$ \\
\hline N & $71.2 \%$ & $3.85 \mathrm{Mbps}$ & $100.0 \%$ & $402 \mathrm{Kbps}$ & $100.0 \%$ \\
¿Thru & $92.5 \%$ & $4.01 \mathrm{Mbps}$ & $104.2 \%$ & $568 \mathrm{Kbps}$ & $141.3 \%$ \\
BTR & $93.1 \%$ & $4.05 \mathrm{Mbps}$ & $105.2 \%$ & $611 \mathrm{Kbps}$ & $152.0 \%$ \\
$B_{\text {avail }}$ & $94.3 \%$ & $4.11 \mathrm{Mbps}$ & $106.8 \%$ & $671 \mathrm{Kbps}$ & $166.9 \%$ \\
\hline
\end{tabular}

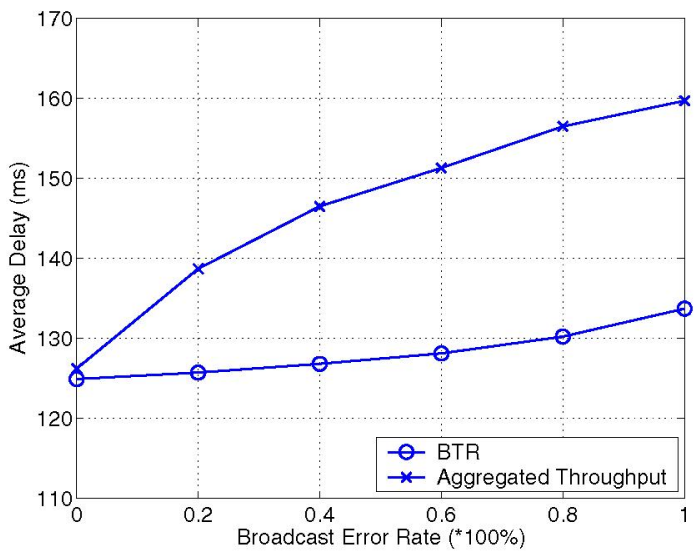

Fig. 6. Delay comparison of $B T R$ and $\sum T h r u$ under different broadcast error rates.

consumption and measurement overhead. The other metrics like $N$ and $\sum$ Thru always rely on the cooperation of neighboring users.

\section{E. Route and Channel Selection in Multi-hop Networks}

In this part, we examine the performance of $\sum$ Thru and $B T R$-based schemes in multi-hop networks. We randomly deploy 20 node pairs in a $1000 \mathrm{~m} \times 1000 \mathrm{~m}$ square area, using one of the two channels to communicate. The maximum hop distance required for any two distinct nodes to communicate is 2. Assuming each node pair carries exponential on/off traffic, we measure the average end-to-end delay under different $B E R$ s. If no neighbor information is successfully received, the latest valid self-observed result is used instead for channel selection. Simulation results in Fig. 6 show that the proposed $B T R$-based scheme significantly outperforms the $\sum$ Thrubased scheme, particularly under high broadcast errors.

\section{CONCLUSION}

In this paper, we propose a new $B T R$-based channel quality metric and a distributed scheme to collaboratively measure $B T R$ across multiple channels with minimum cost and robustness to packet losses. Experimental results show that the proposed scheme achieves significant performance improvements. The proposed algorithms can be implemented using conventional 802.11 hardware with single half-duplex radio interface.

\section{REFERENCES}

[1] N. Jain, S. R. Das, and A. Nasipuri, "A Multi-channel CSMA MAC Protocol with Receiver-based Channel Selection for Multi-hop Wireless Networks," Proc. of International Conference on Computer Communications and Networks'01, Oct. 2001.

[2] R. Prasad, C. Dovrolis, M. Murray, and K. Claffy, "Bandwidth Estimation: Metrics, Measurement Techniques and Tools," IEEE Networks, Vol. 17, Issue 6, Nov.-Dec. 2003.

[3] D. Zheng, J. Zhang, "Protocol Design and Performance Analysis of Opportunistic Multi-Channel Medium Access Control," Arizona State Univ. Tehnical Report, Jan. 2004.

[4] J. So, N. Vaidya, "Multi-channel MAC Protocol for Ad Hoc Networks: Handling Multi-channel Hidden Terminals Using a Single Transceiver," Proc. of MobiHoc'04, May, 2004.

[5] A. Raniwala and T. C. Chiueh, "Architecture and Algorithms for an IEEE 802.11-Based Multi-Channel Wireless Mesh Network," Proc. of IEEE INFOCOM'05, 2005.

[6] Y. C. Tseng, S. L. Wu, C. Y. Lin, and J. P. Shen, "A Multi-channel MAC Protocol with Power Control for Multi-hop Mobile Ad Hoc Networks," Proc. of 21st International Conference on Distributed Computing Systems Workshop, Apr. 2001

[7] F. Cali, M. Conti, and E. Gregori, "Dynamic Tuning of the IEEE 802.11 Protocol to Achieve a Theoretical Throughput Limit," IEEE/ACM Trans. on Networking, Vol. 8, No. 6, Dec. 2000.

[8] G. Bianchi, I. Tinnirello, "Kalman Filter Estimation of the Number of Competing Terminals in an IEEE 802.11 Network," Proc. of IEEE INFOCOM'03, 2003.

[9] IEEE 802.11 Working Group, "Wireless LAN Medium Access Control (MAC) and Physical Layer (PHY) Specifications," Jun. 1999.

[10] C. Guo, Z. Guo, Q. Zhang, and W. Zhu, "A Seamless and Proactive Endto-End Mobility Solution for Roaming Across Heterogeneous Wireless Networks," IEEE JSAC, Vol. 22, No. 5, Jan. 2004

[11] S. Biswas and S. Datta, "Reducing Overhearing Energy in 802.11 Networks by Low-power Interface Idling," Proc. of IEEE IPCCC'04, Apr. 2004.

[12] The VINT Project, "The Network Simulator (version 2), NS-2".

[13] The CMU Monarch Project, "Wireless and Mobility Extension to ns".

[14] J. Zhao, H. Zheng and G. H. Yang, "Distributed Coordination in Dynamic Spectrum Allocation Networks," Proc. of IEEE DySPAN 2005, Nov. 2005. 